\section{Validação de inquérito de risco referido para vigilância em saúde de fatores de risco de doença arterial coronariana em servidores públicos estaduais de Juiz de Fora, Minas Gerais, Brasil}

\author{
Validation of a health survey questionnaire \\ focusing on risk factors for coronary artery disease \\ in a group of public employees in Juiz de Fora, \\ Minas Gerais State, Brazil
}

\author{
1 Instituto de Previdência \\ dos Servidores do Estado \\ de Minas Gerais, \\ Belo Horizonte, Brasil. \\ 2 Departamento de \\ Parasitologia, Instituto \\ de Ciências Biológicas, \\ Universidade Federal \\ de Minas Gerais, \\ Belo Horizonte, Brasil. \\ Correspondência \\ Roberto José Bittencourt \\ Rua Gonçalves Dias 1434 \\ 4 o andar, Belo Horizonte, $M G$ \\ 30140-092, Brasil. \\ bittencourt@ipsemg.mg.gov.br
}

\begin{abstract}
The validity of a health survey questionnaire used in a family health promotion program to identify individuals at risk of developing coronary artery disease (CAD) was studied in a random sample of 297 State public employees in Juiz de Fora, Minas Gerais State, Brazil. The sample was stratified by sex, and subjects were 40 years of age or older. Results obtained in interviews were compared to laboratory and clinical data. We assessed the questionnaire's sensitivity, specificity, and positive and negative predictive values in relation to questions about current or previous history of diabetes, hypertension, smoking, and high cholesterol. Among individuals with at least one altered clinical or laboratory test, $81 \%$ (total sensitivity) were identified by the referred risk inquiry when reporting at least one risk factor. Sheffield and New Zealand tables were used to determine the individuals with increased risk of developing $C A D$ and to assess if they would also be identified by the health survey questionnaire. The sensitivity for identifying this subgroup was $100 \%$.
\end{abstract}

Risk Factors; Coronary Artery Disease; Public Health Survey
Roberto José Bittencourt 1 Sandro Rodrigues Chaves 1 Roberto Campos Amado 2 Valda Franqueira Mendonça 1 Flávio José Fonseca de Oliveira 1 Carlos Maurício de Figueiredo Antunes 2

\section{Introdução}

Em 1999 o Instituto de Previdência dos Servidores de Minas Gerais (IPSEMG), que atende cerca de 470 mil servidores públicos ativos e inativos e seus familiares, lançou o Programa IPSEMG-Família com objetivo de direcionar esforços na prevenção das principais doenças e promover a saúde dos seus beneficiários. O programa é operacionalizado por equipes de profissionais formadas por médico, enfermeiro, odontólogo, psicólogo e assistente social, capazes de abordar de forma sistemática os problemas relacionados à saúde dos segurados cadastrados. Para caracterizar a população de beneficiários do Programa, residentes em diversos municípios do estado, o grupo gestor desenvolveu um inquérito de risco referido (INQ-RR) padrão, denominado Ficha de Saúde, aplicado pelas equipes que realizaram busca ativa dos servidores. Os resultados foram introduzidos em banco de dados de grande porte o que permitiu a determinação da prevalência de diversas doenças e fatores de risco. As pessoas em risco de desenvolver afecções específicas foram identificadas pelas equipes de saúde da família e encaminhadas a exames complementares, monitoramento clínico e participação em oficinas de saúde.

A iniciativa do IPSEMG, por meio de seu programa de saúde da família, de levantar junto a sua população-alvo fatores de risco para 
ocorrência de doenças crônicas, parece inovadora entre as instituições de atenção à saúde no País, considerando que o Ministério da Saúde 1 documenta a carência de informações epidemiológicas relativas aos fatores de risco relacionados a essas doenças - fatores em sua maioria evitáveis com a modificação de estilo de vida, a detecção precoce e o controle oportuno.

No Brasil, segundo dados do Ministério da Saúde 1, as doenças cardiovasculares são a causa de $27,6 \%$ dos óbitos registrados, sendo a causa principal nas regiões Sul e Sudeste. As doenças isquêmicas cardíacas, nessas regiões, respondem por $29,0 \%$ dos óbitos atribuídos a doenças cardiovasculares.

A avaliação inicial do perfil dos segurados atendidos pelo IPSEMG-Família indicava uma prevalência considerável de fatores de risco (tabagismo, hipertensão arterial, hipercolesterolemia, diabetes) associados à doença arterial coronariana (DAC) pelo estudo de Framingham 2. $\mathrm{O}$ risco absoluto de cada segurado é inadequadamente determinado pela análise do efeito isolado destes fatores. Existe a evidência de que estes se combinam em alguns portadores aumentando o risco, que pode ser estimado com auxílio de guias de tratamento em prevenção primária como o de Sheffield 3 e da Nova Zelândia 4 . Esses guias fornecem uma estimativa do risco absoluto por meio da avaliação da taxa de colesterol total pelo HDL combinada com o valor da pressão arterial medida (guia Nova Zelândia 4), ou presença de hipertensão, mesmo em tratamento (guia Sheffield 3 ). Outros fatores que constituem o cálculo são: presença de diabetes, hábito tabagista, sexo e idade. Portanto, um perfil integrado de questões sobre os fatores de DAC, capaz de identificar os indivíduos com risco elevado, deveria ser obtido em um instrumento de vigilância em saúde.

A limitação da utilização da auto-referência de fatores de risco através de um INQ-RR para a correta identificação dos indivíduos em risco, considerando a possibilidade de haver desconhecimento ou desinformação sobre o verdadeiro diagnóstico, era uma preocupação do grupo gestor.

O presente trabalho tem como objetivo determinar a validade do INQ-RR do Programa IPSEMG-Família, Ficha de Saúde, na determinação de pessoas em risco de desenvolver DAC comparado a padrões de análise clínica e laboratorial.

\section{Métodos}

Foi calculada uma amostra de 306 indivíduos da população de 1.514 servidores públicos ca- dastrados no programa IPSEMG-Família de Juiz de Fora, Minas Gerais, com idade acima de 40 anos, completos até o fim de 2001. Para o cálculo foi considerada uma prevalência de $50,0 \%$ dos fatores de risco de DAC e uma precisão de estimativa de $5 \%$ para um intervalo de confiança de $95 \%$. Para compor a amostra, foram sorteados da listagem contida no banco de dados do IPSEMG-Família 81 homens e 351 mulheres, respeitando a distribuição por sexo da população cadastrada. Todos aqueles que efetivamente concordaram em participar assinaram o Termo de Consentimento Livre e Esclarecido. A pesquisa foi aprovada pelo Comitê de Ética em Pesquisa do IPSEMG.

Cada participante foi entrevistado, em julho de 2002, por um integrante da equipe de saúde da família do Programa IPSEMG-Família, que registrou as respostas em um INQ-RR padronizado com perguntas sobre história presente ou anterior de hipertensão arterial, diabetes mellitus, colesterol elevado (hipercolesterolemia) e respectivos tratamentos, e tabagismo. Os participantes foram avaliados clinicamente para medição de pressão arterial, peso e altura. Após as entrevistas, os beneficiários atuantes em instituições localizadas em áreas distintas ou inativos residentes em diversas localidades eram conduzidos a um único laboratório que utilizou métodos confiáveis de rotina para exames de glicemia em jejum, colesterol total e HDL. Os resultados dos exames laboratoriais foram anotados em documentos estruturados, padronizados, distribuídos para as equipes. O manual de instruções fornecido para preenchimento da Ficha de Saúde incluía a restrição a rasuras ou qualquer outro tipo alteração que atingisse a confiabilidade dos dados.

A sensibilidade, a especificidade e os valores preditivos positivo (VPP) e negativo (VPN), com seus respectivos intervalos de confiança, foram calculados para as questões dos fatores de risco isolados.

Foi investigada a validade da utilização de um perfil de risco integrado da Ficha de Saúde que considera combinações de respostas positivas aos fatores, comparando com o padrão de alto risco o fornecido pelas tabelas de Sheffield 3 e da Nova Zelândia 4 . Os indivíduos que não sabiam o nível colesterol sanguíneo foram somados aos que responderam positivamente à presença deste fator 5 . Para a análise da validade da questão sobre diabetes, os que não sabiam foram reunidos ao que responderam negativamente.

O critério-padrão do diagnóstico de hipertensão arterial foi o indivíduo apresentar o valor médio (duas medidas com intervalo de 15 
minutos) da pressão sanguínea sistólica igual ou acima de $140 \mathrm{mmHg}$ ou diastólica igual ou acima de $90 \mathrm{mmHg}$ ou declarar estar em tratamento anti-hipertensivo 3 .

Foram considerados diabéticos os participantes que apresentaram valor de glicemia acima de $126 \mathrm{mg} / \mathrm{dl}$ e/ ou declararam estar em tratamento ${ }^{4}$. Valores de colesterolemia igual ou acima de $200 \mathrm{mg} / \mathrm{dl} \mathrm{e} /$ ou presença de tratamento específico foram utilizados como critério de diagnóstico de hipercolesterolemia 6.

Foram considerados tabagistas as pessoas que afirmavam fumar qualquer quantidade de cigarros no momento ou até um mês anterior à pesquisa 7 .

Os dados foram digitados em dupla entrada em bancos de dados Access e os cálculos estatísticos foram realizados em Stata e Epi-Info. O teste empregado para comparar freqüências foi o qui-quadrado $\left(\chi^{2}\right)$ com correção de Yates considerando o valor $\mathrm{p}$ de 0,05 como limite para significância.

\section{Resultados}

Participaram do estudo 297 segurados cadastrados no IPSEMG-Família, ou seja, 97,0\% da amostra calculada. As perdas deveram-se principalmente a desligamento de funcionários, localização dificultada por viagens e recusas. Os motivos para as recusas foram investigados e não se relacionaram ao objeto da pesquisa.

O percentual de homens, 20,0\% (60/297), e de mulheres, 80,0\% (237/297), não diferiu da população de servidores públicos cadastrados no IPSEMG-Família de Juiz de Fora.

Cento e trinta e sete participantes $(46,0 \%)$ tinham idade entre 40 e 49 anos; 125 (42,0\%), entre 50 e 59 anos; 27 (9,0\%), entre 60 e 69 anos; e 7 (2,0\%), entre 70 e 79 anos. Apenas um participante tinha idade acima de 80 anos. O perfil de escolaridade dos participantes, semelhante ao da população, assegurou a representatividade da amostra (Tabela 1).

\section{Diabetes mellitus}

A história atual ou prévia de diabetes foi positiva em $7,0 \%$ dos participantes, ou seja, 21 do total de 296 respostas válidas (Tabela 2). A distribuição por gênero da referência de diabetes e do diagnóstico clínico-laboratorial não mostrou diferenças significativas.

Considerando diabéticos aqueles que estavam em tratamento no momento da pesquisa e/ou que apresentavam valores de glicemia acima de $126 \mathrm{mg} / \mathrm{dl}$, a prevalência foi de $6,0 \%$
Tabela 1

Escolaridade dos participantes amostrados e da população de segurados do Instituto de Previdência dos Servidores de Minas Gerais (IPSEMG) de Juiz de Fora, Minas Gerais, Brasil.

\begin{tabular}{lrrrr}
\hline \multirow{2}{*}{ Escolaridade } & \multicolumn{2}{c}{ Amostra } & \multicolumn{2}{c}{ População } \\
& $n$ & $\%$ & $n$ & $\%$ \\
\hline 1。 Grau & 71 & 23,9 & 337 & 22,3 \\
2ㅇ Grau & 73 & 24,6 & 375 & 24,8 \\
3ㅇ Grau & 99 & 33,3 & 525 & 34,7 \\
Pós-graduação & 54 & 18,2 & 277 & 18,3 \\
Total & 297 & 100,0 & 1.514 & 100,0 \\
\hline
\end{tabular}

$\mathrm{P}=0,93$

Tabela 2

Prevalência dos fatores de risco para doença arterial coronariana (DAC), determinada pela resposta afirmativa (+) à Ficha de Saúde e pelo diagnóstico clínico-laboratorial.

\begin{tabular}{lcccc}
\hline Fator de risco & $\begin{array}{c}\text { Masculino } \\
\%\end{array}$ & $\begin{array}{c}\text { Feminino } \\
\%\end{array}$ & \multicolumn{2}{c}{ Total } \\
& & & & $n$ \\
\hline Diabetes & 8,0 & 7,0 & 7,0 & 296 \\
Resposta + & 8,0 & 5,0 & 6,0 & 297 \\
Diagnóstico & & & & \\
Hipertensão & 30,0 & 34,0 & 33,0 & 296 \\
Resposta + & $57,0 \mathrm{a}$ & $35,0 \mathrm{a}$ & 39,0 & 297 \\
Diagnóstico & & & & \\
Hipercolesterolemia & $18,0 \mathrm{~b}$ & $37,0 \mathrm{~b}$ & 33,0 & 296 \\
Resposta + & 48,0 & 53,0 & 52,0 & 297 \\
Diagnóstico & & & & \\
Tabagismo & $37,0 \mathrm{c}$ & $22,0 \mathrm{c}$ & 25,0 & 297 \\
Resposta + & & & & \\
\hline
\end{tabular}

Teste do $\chi^{2}$ : axa: $p=0,003 ; b x b ; p=0,016 ; c x c: p=0,035$.

(17/296). Destes, apenas um respondeu negativamente ao INQ-RR, 14 referiram tratamento e em dez o nível glicêmico estava sob controle, abaixo de $126 \mathrm{mg} / \mathrm{dl}$ (Figura 1).

Oito (3,0\%) participantes desconheciam seu diagnóstico de diabetes, todos com resultado laboratorial de glicemia negativo. Estes participantes foram somados aos que responderam negativamente à questão durante a entrevista para cálculo de validação.

A Tabela 3 mostra os dados utilizados nos cálculos de validação das questões sobre os fatores de risco isolados. A sensibilidade da ques- 
Figura 1

Distribuição dos diabéticos, hipertensos e hipercolesterolêmicos de acordo com conhecimento prévio do diagnóstico, referência de tratamento e controle dos níveis de glicemia, pressórico e de colesterol sérico.

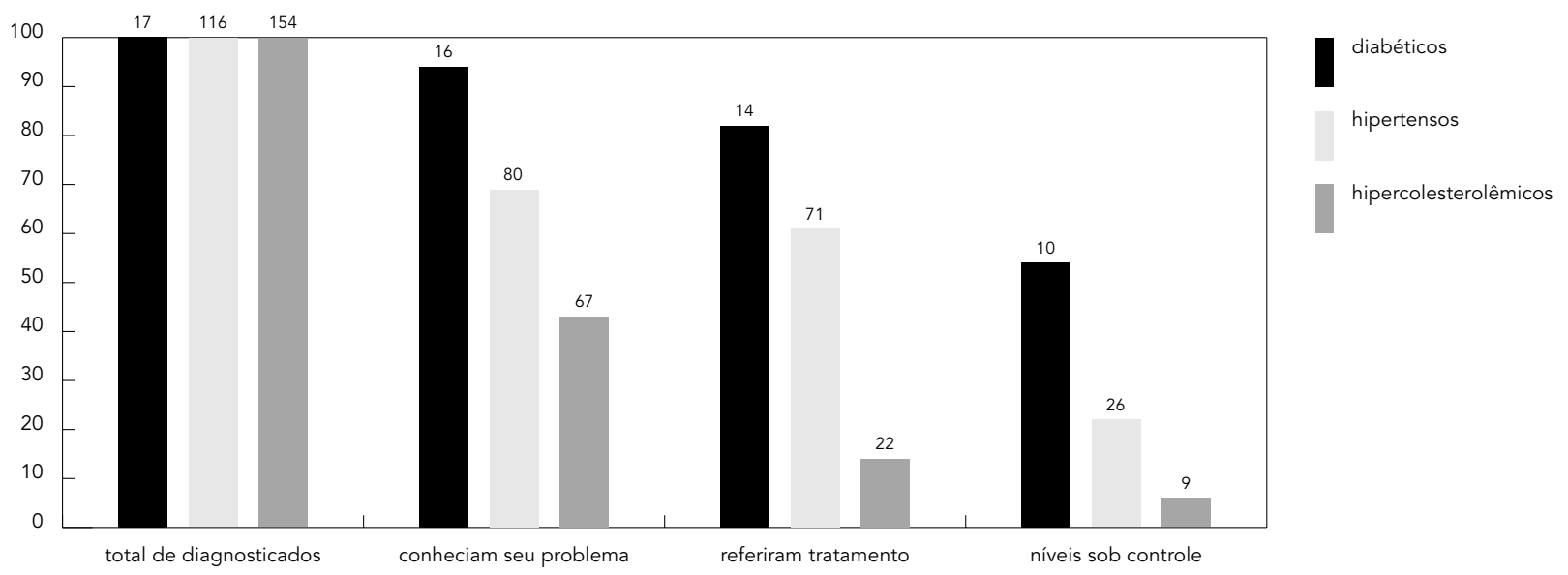

Tabela 3

Comparação das respostas ao inquérito de risco referido em relação ao diagnóstico clínico-laboratorial e validade (\%) das questões isoladas.

\begin{tabular}{|c|c|c|c|c|c|c|c|}
\hline \multirow[t]{2}{*}{ Diagnóstico clínico } & \multicolumn{3}{|c|}{ Respostas } & \multirow{2}{*}{$\begin{array}{l}\text { Sensibilidade } \\
\text { (IC95\%) }\end{array}$} & \multirow{2}{*}{$\begin{array}{l}\text { Especificidade } \\
\text { (IC95\%) }\end{array}$} & \multirow{2}{*}{$\begin{array}{c}\text { VPP } \\
\text { (IC95\%) }\end{array}$} & \multirow{2}{*}{$\begin{array}{c}\text { VPN } \\
\text { (IC95\%) }\end{array}$} \\
\hline & Sim & Não & Não sabe & & & & \\
\hline \multicolumn{8}{|l|}{ Diabetes } \\
\hline+ & 16 & 1 & 0 & $94,1(69,2-99,7)$ & $98,2(95,6-99,3)$ & $76,1(52,4-90,9)$ & $99,6(97,7-10,0)$ \\
\hline- & 5 & 266 & 8 & & & & \\
\hline \multicolumn{8}{|l|}{ Hipertensão } \\
\hline+ & 80 & 36 & 0 & $69,0(59,6-77,0)$ & $90,0(84,4-93,8)$ & $81,6(72,3-88,5)$ & $81,8(75,6-86,8)$ \\
\hline- & 18 & 162 & 0 & & & & \\
\hline \multicolumn{8}{|l|}{ Hipercolesterolemia } \\
\hline+ & 66 & 76 & 11 & $50,3(42,2-58,5)$ & $76,2(68,2-82,8)$ & $69,4(59,8-77,6)$ & $58,9(51,4-66,0)$ \\
\hline- & 33 & 109 & 1 & & & & \\
\hline
\end{tabular}

VPP = valor preditivo positivo; VPN = valor preditivo negativo.

tão sobre diabetes foi de 94,1\% (IC95\% de 69,2 a 99,7); a especificidade, de $98,2 \%$ (IC de 95,6 a 99,3); o VPP, de $76,1 \%$ (IC de 52,4 a 90,9 ); e o VPN, de $99,6 \%$ (IC de 97,7 a 100,0).

\section{Hipertensão}

A história de hipertensão arterial sistêmica atual ou prévia foi referida por 33,0\% (98/296) dos participantes (Tabela 2). Houve somente uma recusa de resposta. Nenhum participan- te respondeu não saber sobre seu nível pressórico.

A hipertensão, pelo critério clínico de referir tratamento específico ou apresentar valores médios medidos de pressão sistólica acima ou igual a 140mmHg ou diastólica acima ou igual a 90mmHg, teve prevalência de 39,0\% (117/297).

Não houve diferença significativa entre os sexos na referência da história atual ou prévia de hipertensão $\left(\chi^{2}=0,18 ; \mathrm{p}=0,67\right)$. O diagnóstico clínico desse fator, a pressão elevada ou a 
referência de tratamento foram mais freqüentes entre os homens $\left(\chi^{2}=8,51 ; p<0,05\right)$.

Cerca de um terço dos hipertensos desconhecia o problema e $60,0 \%$ referiram tratamento. O controle da pressão foi observado em um terço do grupo (Figura 1).

A sensibilidade da questão sobre hipertensão arterial foi de $69,0 \%$ (IC95\% de 59,6 a 77,0); a especificidade, de $90,0 \%$ (IC95\% de 84,4 a 93,8); o VPP, de $81,6 \%$ (IC95\% de 72,3 a 88,5 ); e o VPN, de $81,8 \%$ (IC95\% de 75,6 a 86,8 ). Estes dados podem ser visualizados na Tabela 3 .

\section{Hipercolesterolemia}

Responderam afirmativamente a questão sobre história atual ou prévia de colesterol elevado 33,0\% (99/296) dos respondentes (Tabela 2). Os homens referiram significativamente menos a hipercolesterolemia do que as mulheres, porém, para ambos, a referência subestima a prevalência diagnosticada, que é semelhante entre os gêneros.

O diagnóstico clínico indicou a prevalência geral de 52,0\% (154/297) de hipercolesterolêmicos. Menos da metade dos diagnosticados conhecia seu problema, somente $14,0 \%$ referiram tratamento específico e metade conseguiu o controle do colesterol ao nível desejável (Figura 1).

Não souberam responder à questão 4,0\% (12/296), dos quais um apresentou valor de colesterol total abaixo de $200 \mathrm{mg} / \mathrm{dl}$ e 11 , acima de $200 \mathrm{mg} / \mathrm{dl}$. Estes participantes foram somados aos que responderam positivamente à questão durante a entrevista para cálculo de validação.

Os dados para o cálculo validade da questão sobre colesterol elevado estão descritos na Tabela 3. A sensibilidade da questão foi de $50,3 \%$ (IC95\% de 42,2 a 58,5); a especificidade, de $76,2 \%$ (IC95\% de 68,2 a 82,8); o VPP, de 69,4\% (IC95\% de 59,8 a 77,6 ); e o VPN, de $58,9 \%$ (IC95\% de $51,4$ a 66,0$)$.

\section{Tabagismo}

A prevalência de tabagismo atual ou suspenso menos de um mês antes foi de $25,5 \%$, nunca ter fumado foi a resposta de $49,0 \%$ e $25,5 \%$ abandonaram o hábito de fumar mais de um mês antes (Tabela 2).

O tabagismo atual ou suspenso menos de um mês antes foi relatado com maior freqüência por homens $\left(\chi^{2}=4,46 ; \mathrm{p}=0,035\right)$.

\section{Validação do perfil de risco da Ficha de Saúde}

\section{Comparada aos padrões}

Considerando a tabela de Sheffileld 3 , o risco acima de $15,0 \%$ ou maior de desenvolver DAC em dez anos foi observado em 9,0\% (27/297) dos participantes. O guia da Nova Zelândia 4 aponta 6,0\% (19/297) de participantes em risco de DAC em cinco anos.

A composição do perfil de risco da Ficha de Saúde incluiu os participantes que forneceram respostas válidas. Somente não foi possível analisar uma segurada, de 79 anos, que não respondeu às questões, tendo realizado os exames laboratoriais que indicaram a presença de risco elevado segundo os guias.

A Tabela 4 mostra que a maior sensibilidade, característica desejável do INQ-RR avaliado, foi alcançada pelo perfil que considera alvo de acompanhamento pela equipe de saúde da família o entrevistado que refere positivamente a presença de qualquer um dos quatro fatores de risco. Comparado aos padrões de Sheffield 3 e Nova Zelândia 4 , esse perfil tem sensibilidade de identificar 100,0\% dos segurados com risco elevado. A especificidade respectiva aos padrões foi de $37,0 \%$ e de $31,0 \%$; o VPP, de $12,0 \%$ e $10,0 \%$; e o VPN, de $100,0 \%$ e $100,0 \%$.

\section{Comparada aos resultados \\ clínico-laboratoriais}

A capacidade da Ficha de Saúde de identificar os indivíduos que apresentavam pelo menos um exame clínico-laboratorial alterado (glicemia, colesterolemia ou pressão arterial medida) foi descrita na Tabela 5 . A resposta positiva a pelo menos um fator de risco (inclusive tabagismo) da Ficha de Saúde apresentou sensibilidade de $80,9 \%$ (IC95\% de 75,6 a 85,3) para identificar esses participantes. A especificidade desse perfil foi de $84,4 \%$ (IC95\% de 71,2 a 82,3 ); o VPP, de $96,7 \%$ (IC95\% de 93,3 a 98,4); e o VPN, de $44,2 \%$ (IC95\% de 34,2 a 54,7 ).

Dos 86 participantes que não referiram nenhum fator de risco em resposta ao INQ-RR, seis têm hipertensão pelo exame clínico, 34 têm colesterol total igual ou acima de $200 \mathrm{mg} / \mathrm{dl}$ e oito têm ambos os fatores alterados.

\section{Discussão}

A estratégia da utilização da entrevista para coleta de informações na população de servidores públicos estaduais em Juiz de Fora foi bem 
Tabela 4

Comparação de combinações de respostas positivas ao inquérito de risco referido em relação à estimativa de risco pelos guias Sheffield e Nova Zelândia.

\begin{tabular}{|c|c|c|c|c|}
\hline \multirow[t]{3}{*}{ Respostas positivas } & \multicolumn{4}{|c|}{ Padrões } \\
\hline & \multicolumn{2}{|c|}{ Sheffield } & \multicolumn{2}{|c|}{ Nova Zelândia } \\
\hline & Positivo & Negativo & Positivo & Negativo \\
\hline \multicolumn{5}{|l|}{ Dois fatores } \\
\hline+ & 15 & 54 & 7 & 62 \\
\hline- & 12 & 215 & 11 & 216 \\
\hline \multicolumn{5}{|l|}{ Três fatores } \\
\hline+ & 6 & 7 & 5 & 8 \\
\hline- & 21 & 262 & 13 & 270 \\
\hline \multicolumn{5}{|l|}{ Um ou mais* } \\
\hline+ & 26 & 184 & 18 & 192 \\
\hline- & 0 & 86 & 0 & 86 \\
\hline
\end{tabular}

* Validade (IC95\%) padrão Sheffield: Sensibilidade $=100,0 \%(84,0-100,0)$; Especificidade $=31,9 \%(26,4-37,8) ; \mathrm{VPP}=12,4 \%(8,4-17,8) ; \mathrm{VPN}=100,0 \%(94,7-100,0)$ * Validade (IC95\%) padrão Nova Zelândia: Sensibilidade $=100,0 \%(78,1-100,0)$; Especificidade $=30,9 \%(25,6-36,8) ; \operatorname{VPP}=8,6(5,3-13,4) ; \operatorname{VPN}=100,0(94,7-100,0)$

Tabela 5

Comparação do perfil de resposta positiva a pelo menos um fator de risco da Ficha de Saúde com a positividade dos exames clínico-laboratoriais.

\begin{tabular}{lccc}
\hline & \multicolumn{3}{c}{ Resultados clínico-laboratoriais } \\
& Positivos & Negativos & Total \\
\hline Ficha de Saúde* & & & \\
+ & 203 & 7 & 210 \\
- & 48 & 38 & 86 \\
Total & 251 & 45 & 296 \\
\hline
\end{tabular}

* Validade (IC95\%) Sensibilidade $=80,9 \%(75,6-85,3)$; Especificidade $=84,4 \%$ $(71,2-82,3) ; \mathrm{VPP}=96,7 \%(93,3-98,4) ; \mathrm{VPN}=44,2 \%(34,2-54,7)$

sucedida. A taxa de resposta foi alta e somente uma participante não respondeu ao INQ-RR, tendo realizado os exames e assinado o Termo de Consentimento Livre e Esclarecido. Seriam esperados valores maiores de não-resposta caso o INQ-RR fosse enviado pelo correio ou aplicado por telefone. Haveria também menor possibilidade de negociação quanto a local e tempo e de explorar questões que requerem explicações do entrevistador 8 .

Uma revisão da literatura nacional e internacional indica que a validade de questões isoladas para detectar fatores de risco de DAC é relativamente limitada em diversas populações, independentemente de fatores externos capazes de influenciar a resposta correta, como gênero, faixa etária ou categoria profissional. Esse fato nos motivou a avaliar a combinação de questões do inquérito que fosse mais sensível, permitindo identificar beneficiários em risco, que se tornariam alvo da atenção primaria à saúde.

Segundo dados da Sociedade Brasileira de Diabetes $9,46,5 \%$ dos pacientes diabéticos de 30 a 69 anos desconhecem seu diagnóstico. Goldenberg et al. 10 mostraram que a prevalência de diabetes mellitus, auto-referida por $4,7 \%$ dos participantes do INQ-RR domiciliar realizado em São Paulo, foi subestimada em comparação ao diagnóstico da doença observado em $9,7 \%$ da amostra. Em nossa pesquisa, os diabéticos pareciam bem mais conscientes de sua saúde, fato demonstrado pela sensibilidade de 96,0\% da questão. Mesmo aqueles que afirmaram ignorar seu diagnóstico apresentaram resultado negativo ao exame de glicemia em laboratório.

A proporção de 31,0\% de hipertensos diagnosticados que desconheciam seu problema é semelhante à descrita por Piccini \& Victora 11 , de $32,0 \%$, em Pelotas, Rio Grande do Sul, e um pouco menor do que a observada por Kirkland et al. 12 entre canadenses de 55 a 74 anos de idade, de $38,0 \%$.

Os homens canadenses, assim como os da amostra de Juiz de Fora, apresentaram menor consciência da hipertensão do que as mulheres, com proporções respectivas de $43,0 \%$ e 33,0\% de desconhecimento do diagnóstico ${ }^{12}$. Na Suécia, as mulheres foram as que apresentaram menor conhecimento prévio da hipertensão; a sensibilidade da questão para esse gênero foi de apenas $29,0 \%$ e, entre os homens, de $69,0 \% 5$.

$O$ relato de hipertensão foi validado na Espanha por Tormo et al. 13, que compararam a informação de pacientes a um INQ-RR com registros médicos de diagnóstico ou uso de medicação anti-hipertensiva. Os autores encontraram valores de $63,5 \%$ de sensibilidade e $91,4 \%$ de especificidade para a questão. A sensibilidade foi maior entre as mulheres e entre as pessoas com menor nível de instrução. Valores próximos foram observados em Juiz de Fora $69,0 \%$ e $90,0 \%$, respectivamente para sensibilidade e especificidade, dessa questão isolada no inquérito.

Em um estudo realizado por Dioguardi et al. 14 entre 1.395 médicos voluntários do Estado de São Paulo, com idade entre 24 e 77 anos, $75,9 \%$ dos 333 participantes diagnosticados com hipertensão na época da pesquisa não sabiam que estavam com níveis pressóricos elevados; $44,1 \%$ que apresentavam colesterolemia 
acima de $240 \mathrm{mg} /$ dl não referiram antecedente de positividade, portanto não sabiam ser hipercolesterolêmicos. Essa pesquisa indica que o desconhecimento da hipertensão não se restringe a grupos, a priori, leigos em diagnósticos clínicos.

O desconhecimento da hipercolesterolemia foi observado em $65,0 \%$ dos canadenses, nível mais elevado do que os $57,0 \%$ de Juiz de Fora. Os pesquisadores usaram a mesma concentração-limite de $200 \mathrm{mg} / \mathrm{dl}$ de colesterol sérico para diagnóstico laboratorial 12 .

A validade da referência de hipercolesterolemia entre adultos americanos foi investigada por Natarajan et al. 6 , que descreveram valores de $51,0 \%$ de sensibilidade e $89,0 \%$ de especificidade, muito próximos aos encontrados no presente estudo. Os autores alertaram para a necessidade de estudos futuros da mesma natureza, a fim de alicerçar pesquisas populacionais baseadas na referência.

O valor do relato do tabagismo como medida indireta de exposição em estudos populacionais foi validado pela comparação com a medida de cotinina sérica, o maior metabólito plasmático da nicotina. Há correlação direta entre os níveis de cotinina sérica e o consumo de cigarros (atual/há mais de um mês/nunca) referido pelos entrevistados de diversas áreas, idades e grupos sócio-econômicos 15. No presente estudo consideramos suficiente a resposta do entrevistado e não realizamos a dosagem de cotinina sérica.

Os participantes da pesquisa que apresentavam risco absoluto de DAC acima de $15,0 \%$ estimado pelos padrões Sheffield 3 e Nova Zelândia 4 foram corretamente identificados pela Ficha de Saúde, ou seja, 100,0\%. Seria inadmissível que essas pessoas fossem perdidas no processo de vigilância em saúde e nas ações de prevenção, pois, segundo estes parâmetros internacionais, seria o grupo de risco mais grave.

Possivelmente a propriedade mais relevante da Ficha de Saúde, tenha sido a integração dos quatro fatores de risco para DAC em um mesmo inquérito, quando encontramos a sensibilidade total de $80,9 \%$, considerada alta, tratando-se de um instrumento não-invasivo de diagnóstico, o que recomenda seu uso para vigilância em saúde. Além disso, o VPP de 96,7\% da Ficha de Saúde demonstra que na população Juiz de Fora, com elevada prevalência de fatores diagnosticados, poucos inquéritos negativos seriam alvo de maior atenção primária. Portanto, todos os segurados que relatam ter um ou mais fatores de risco serão monitorados clinicamente e convidados a participar de oficinas de saúde.
Em função dos resultados encontrados, a prática da medida da pressão arterial em todos os beneficiários atendidos também deverá ser adotada pelas equipes de saúde da família e, considerando-se a questão custo-benefício dos exames de colesterol total e fração HDL, estes deverão ser solicitados para todos os beneficiários com idade igual ou acima de quarenta anos.

O INQ-RR tem sido utilizado com sucesso pelo Centro de Controle e Prevenção de Doenças (CDC) dos Estados Unidos como instrumento de coleta de informações para monitoramento da saúde da população.

O National Health Interview Survey 16, que constitui a principal fonte de informação sobre a saúde dos cidadãos americanos, vem sendo conduzido desde 1957 e seus dados são divulgados anualmente pela instituição. Os dados provêm de entrevistas pessoais dirigidas a indivíduos adultos, moradores de residências sorteadas em amostra representativa da população de todo o país. A partir de 1997, com o objetivo de acelerar a aplicação do INQ-RR, as respostas passaram a ser lançadas diretamente no computador pelo entrevistador.

Em 1984, o CDC iniciou o Behavioral Risk Factor Surveillance System (BRFSS) 17 em 15 estados americanos, com objetivo de monitorar, em níveis estadual e regional, a prevalência entre adultos dos principais comportamentos de risco associados a morbidade e mortalidade prematura. A partir de 1994 todos os estados passaram a conduzir o BRFSS. As entrevistas são realizadas por telefone e os dados totalizados mensalmente. As informações provenientes do survey têm sido utilizadas pelos departamentos de saúde para planejar, iniciar, apoiar e avaliar programas de promoção à saúde e prevenção de doenças.

Está em discussão no Ministério da Saúde uma proposta de implantação do monitoramento em nível nacional das doenças não transmissíveis e seus fatores de risco ${ }^{1}$. Entre outras estratégias, encontra-se a realização de INQ-RR por domicílio nacional a cada cinco anos ou menor intervalo, caso necessário em locais específicos. O conhecimento derivado do INQ-RR fornecerá subsídios aos programas de prevenção e controle das doenças no sentido de direcioná-los e redirecioná-los para grupos mais vulneráveis, além de orientar o enfoque de políticas e ações educativas, legislativas e econômicas, com conseqüente aumento de eficiência e efetividade.

A análise do presente estudo sugere a possibilidade de as próprias equipes de saúde da família realizarem o INQ-RR, tornando-o um instrumento ágil e agregando informação no 
local onde ela é capturada. Desta forma, a realização e a análise do INQ-RR poderá ser a ação estruturante das equipes de saúde da família, induzindo-as a uma intervenção pró-ativa.

O INQ-RR sobre fatores de risco de DAC, agregado a outras questões relacionadas a doenças crônicas evitáveis e prevalentes na população de servidores públicos de Minas Gerais, deverá ser utilizado anualmente como informação básica sobre a saúde e o comportamento de risco dos segurados do IPSEMG. A utilização do INQ-RR em toda a população de servidores públicos do estado, bem como a avaliação de dados de morbi-mortalidade da população atendida pelo IPSEMG, integrada ao nível central pelo sistema de Data Warehouse (Armazém de Dados), fornecerá dados ao grupo gestor para planejamento e avaliação de programas de prevenção e diagnóstico precoce de diversas afecções, conduzidos pelas equipes de saúde da família. Essa conduta segue uma tendência apontada por Choi 18 para a vigilância epidemiológica do século 21 - o monitoramento dos indicadores de saúde e fatores de risco como base para estratégias de intervenção. No entanto, novas perguntas se fazem necessárias:

- Em quantos indivíduos serão evitadas as doenças rastreadas nesse tipo de abordagem?

- Em quantos indivíduos serão identificadas precocemente as doenças rastreadas nesse tipo de abordagem?

- Qual o impacto dessas medidas de prevenção e promoção de saúde na qualidade de vida da população de risco?

- Quantos anos de vida útil serão agregados a essa população?

- Qual o impacto econômico no sistema de saúde, quando é possível abordar ativamente os risco de adoecimento?

- O instrumento, INQ-RR, é válido em populações com características distintas dos servidores públicos de Minas Gerais?

Esta pesquisa constatou que os servidores públicos de Juiz de Fora têm um perfil de esco- laridade elevado, com cerca de 53,0\% dos cadastrados no programa IPSEMG-Família apresentando graduação ou pós-graduação. As mulheres constituem a maioria dos servidores cadastrados porque o programa iniciou seu trabalho entre os funcionários da Secretaria de Educação.

Esse perfil de elevada escolaridade e maioria feminina se assemelha ao dos demais servidores públicos estaduais brasileiros, segundo dados do Instituto de Pesquisa Econômica Aplicada (IPEA) 19. Em levantamento realizado pelo órgão, considerando dados médios da Pesquisa Nacional por Amostragem Domiciliar de 1997 a 1999, as mulheres são maioria entre os servidores, $67,0 \%$ do total. Destas, $49,0 \%$ são docentes e 14,2\% são não-docentes com nível superior. As docentes provavelmente têm em sua maioria nível superior, mas esse dado não é explicitado no documento.

Considerando o perfil especial dos servidores públicos estaduais, é possível inferir que o nível de desconhecimento de fatores de risco que envolvem medidas laboratoriais tende a aumentar entre pessoas de menor escolaridade. No entanto, se as equipes de saúde da família considerarem como alvo de abordagem da atenção primária à saúde os respondentes que desconhecem formalmente seu diagnóstico, a validade do INQ-RR para DAC pouco se altera entre as populações de menor nível educacional.

As demais questões não podem ser respondidas no escopo dessa pesquisa, mas, com os resultados obtidos, nos quais encontramos a sensibilidade total de $80,9 \%$, provamos que o INQ-RR constitui um instrumento útil e promissor, na medida em que os beneficiários do IPSEMG com risco de DAC serão identificados precocemente e acompanhados ativamente. Parece-nos que essa abordagem é uma alternativa viável à situação atual, na qual o sistema de saúde só é acionado após o aparecimento de um evento cardiovascular, gerando os altíssimos índices de morbi-mortalidade encontrados no Brasil. 


\section{Resumo}

Foi investigada a validade da Ficha de Saúde, inquérito de risco referido (INQ-RR) do Programa IPSEMGFamília, na identificação de pessoas em risco de desenvolver doença arterial coronariana (DAC) numa amostra aleatória, estratificada por sexo, de 297 segurados com idade igual e superior a 40 anos, de Juiz de Fora, Minas Gerais, Brasil. Os resultados obtidos em entrevistas realizadas pelas equipes de saúde da família foram comparados com dados de exames clínicolaboratoriais. Foram avaliados a sensibilidade, a especificidade e os valores preditivos positivos e negativos das questões sobre história atual ou prévia de diabetes mellitus, hipertensão arterial, colesterol elevado e tabagismo. Dentre os participantes que apresentavam pelo menos um exame clínico-laboratorial alterado, 81,0\% (sensibilidade total) foram identificados pelo inquérito de risco referido ao declarar ter pelo menos um fator de risco. Os padrões de Sheffield e da Nova Zelândia foram utilizados para determinar os segurados com risco elevado de adquirir doença coronariana e avaliar se estes seriam também identificados pela Ficha de Saúde. A sensibilidade para identificar esse subgrupo de pacientes foi de 100,0\%.

Fatores de Risco; Doença da Artéria Coronária; Vigilância em Saúde Pública

\section{Colaboradores}

R. J. Bittencourt participou na coordenação e formulação da pesquisa. S. R. Chaves colaborou na formulação da correlação entre os fatores de risco cardiovascular e o padrão internacional adotado na pesquisa. R. C. Amado contribuiu com a formulação do desenho metodológico do estudo, em especial, da validação estatística das amostras. V. F. Mendonça colaborou na coordenação das equipes de campo para a coleta dos dados. F. J. F. Oliveira formulou a utilização das Fichas de Saúde como fonte de identificação dos fatores de risco. C. M. F. Antunes participou da consolidação do desenho final da pesquisa.

\section{Agradecimentos}

Aos órgãos financiadores Conselho Nacional de Desenvolvimento Científico e Tecnológico (CNPq) e Instituto de Previdência dos Servidores de Minas Gerais (IPSEMG). À bolsista Regiane Carla Ferreira e aos coordenadores do programa IPSEMG-Família em Juiz de Fora, Dr. Osvaldo Reis de Andrade Santos e Dra. Teresa Cristina Naya Loures, pela valiosa contribuição. Aos profissionais das equipes de saúde da família de Juiz de Fora pelo empenho e pela qualidade no trabalho desenvolvido: A Andrade, M Araújo, C Basílio, M Bogado, J Brandão, M Brandi, M Campos, C Cardoso, V Castellães, J Castro, S Castro, A Cláudio, R Cunha, J Damasceno, A David, B Dutra, R Fabrino, M Falcão, A Fávero, R Fernandes, S Ferraz, T Ferreira, M Freitas, M Gravina, C Heleno, R Herédia, J Júnior, N Júnior, R Lanziotti, F Lima, W Louro, J Martins, V Martins, R Moreira, C Morandi, A Mussi, H Nheme, F Nunes, J Oliveira, U Ormundo, A Paiva, E Paiva, A Pimenta, D Queirós, E Rauen, H Rezende, C Ribeiro, P Ronzani, M Ruback, F Sales, J Sampaio, M Silva, W Silveira, M Simões, L Teixeira, A Thomaz, B Tostes, C Vale, VVieira, G Vaz, M Zampier.

\section{Referências}

1. Ministério da Saúde. Proposta de monitoramento dos agravos não transmissíveis e seus fatores de risco. http://www.funasa.gov.br/epi/intransmi/ epi_intransmi_00.htm (acessado em 03/Mai/2002).

2. Wilson PWF. Established risk factors and coronary artery disease: The Framinghan Study. Am J Hypertens 1994; 7 Suppl:7S-12S.

3. Wallis EJ, Ramsay LE, Haq IU, Ghahramani P, Jackson PR, Rowland-Yeo K, et al. Coronary and cardiovascular risk estimation for primary prevention: validation of a new Sheffield table in the 1995 Scottish health survey population. Br Med J 2000; 320:671-6.

4. New Zealand Guidelines Group. The management of mildly raised blood pressure in New Zealand. Estimation of cardiovascular risk for men and women. http://www.nzgg.org.nz/library/gl_ complete/bloodpressure/table1.cfm (acessado em 18/Abr/2002).

5. Johansson J, Hellénius ML, Elofosson S, Krakau I. Self-Report as a selection instrument in screening for cardiovascular disease risk. Am J Prev Med 1999; 16:322-4. 
6. Natarajan S, Lipsitz SR, Nietert PJ. Self report of high cholesterol. Determinants of validity in U.S. adults. Am J Prev Med 2002; 23:13-21.

7. National Cholesterol Education Program. Executive summary of the third report of the National Cholesterol Education Program (NCEP) expert panel on detection, evaluation, and treatment of high blood cholesterol in adults (Adult Treatment Panel III). JAMA 2001; 285:2487-97.

8. Veale B. Questionnaire design and surveys. Aust Fam Physician 1998; 27:499-502.

9. Sociedade Brasileira de Diabetes. Prevalência do diabetes mellitus no Brasil. http://www.diabetes. org.br/Diabetes/estatisticas/Estat2.html (acessado em 11/Jun/2002).

10. Goldemberg P, Franco LJ, Pagliaro H, Silva RS, Santos CA. Diabetes mellitus auto-referido no município de São Paulo: prevalência e desigualdade. Cad Saúde Pública 1996; 12:37-45.

11. Piccini RX, Victora CG. How well is hypertension managed in the community? A population-based survey in a Brazilian city. Cad Saúde Pública 1997; 13:595-600

12. Kirkland AS, MacLean DR, Langille DB, Joffres MR, MacPherson KM, Andreou P. Knowledge and awareness of risk factors for cardiovascular disease among Canadians 55 to 74 years of age: results from the Canadian Heart Health Surveys, 19861992. Can Med Assoc J 1999; 161 Suppl:10S-6S

13. Tormo MJ, Navarro C, Chirlaque MD, Barber X. Validation of self diagnosis of high blood pressure in a sample of the Spanish EPIC cohort: overall agreement and predictive values. EPIC Group of Spain. J Epidemiol Community Health 2000; 54 : 221-6.
14. Dioguardi GS, Pimenta J, Knoplich J, Ghorayeb N, Ramos LR, Gianini SD. Fatores de risco para doenças cardiovasculares em médicos. Dados preliminares do Projeto VIDAM da Associação Paulista de Medicina. Arq Bras Cardiol 1994; 62:383-8.

15. Vartiainen E, Seppälä T, Lillsund P, Puska P. Validation of self reported smoking by sorum cotinine measurement in a community-based study. J Epidemiol Community Health 2002; 56:167-70.

16. Centers for Disease Control and Prevention. National Health Interview Survey. http://ftp.cdc. gov/pub/Health_Statistics/NCHS/Dataset_Docu mentation/NHIS/2001/srvydesc.pdf http://www. cdc.gov/nchs/nhis.html (acessado em 07/Jun/ 2002).

17. Centers for Disease Control and Prevention. Behavioral risk factor surveillance system. About the BRFSS. http://www.cdc.gov/brfss/about.htm (acessado em 07/Jun/2002).

18. Choi BCK. Perspectives on epidemiologic surveillance in the 21st century. Chronic Dis Can 1998; 19:145-51.

19. Pinheiro SS, Sugahara T. Perfil dos funcionários públicos ativos nas áreas federal, estadual e municipal - comparação de bases disponíveis: RAIS, PNAD e SIAPE. Outubro de 2001. http://www. ipea.gov.br/pub/td/td_2001/td_0837.pdf (acessado em 18/Dez/2002).

Apresentado em 03/Abr/2003

Versão final reapresentada em 16/Out/2003

Aprovado em 11/Nov/2003 